
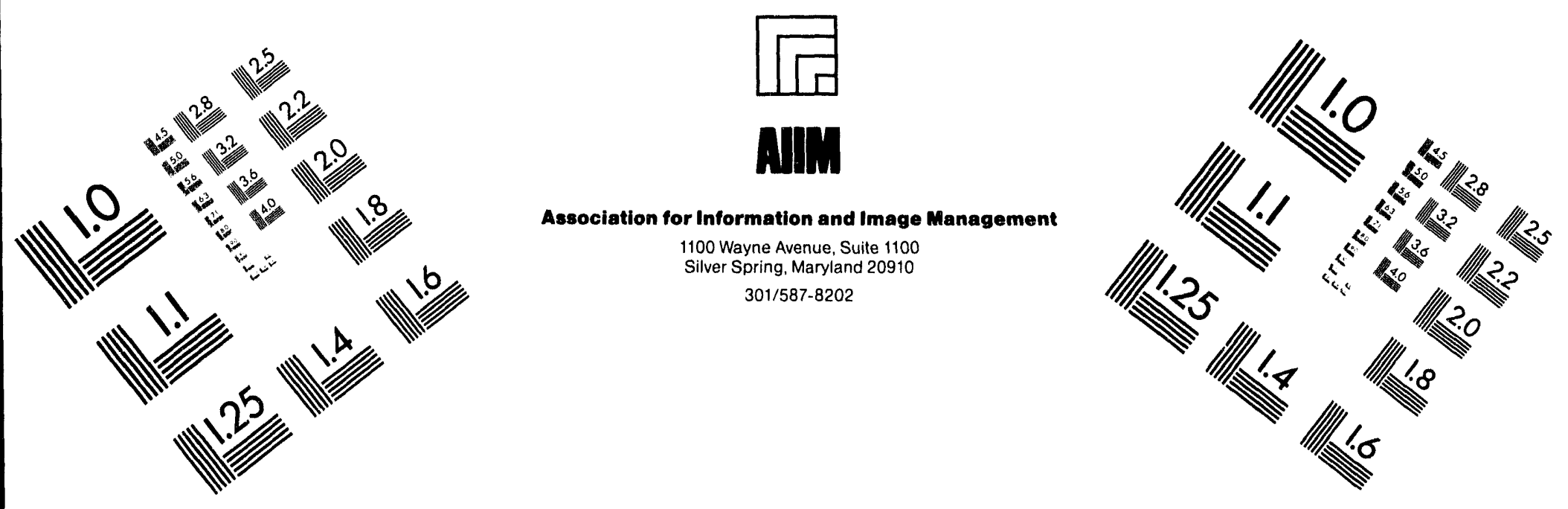

Centimeter

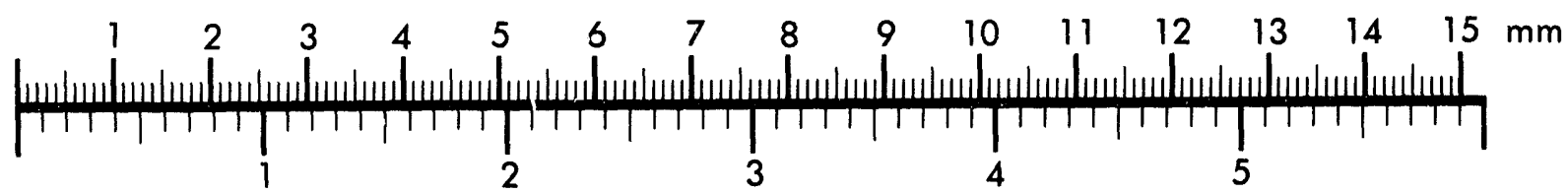
Inches
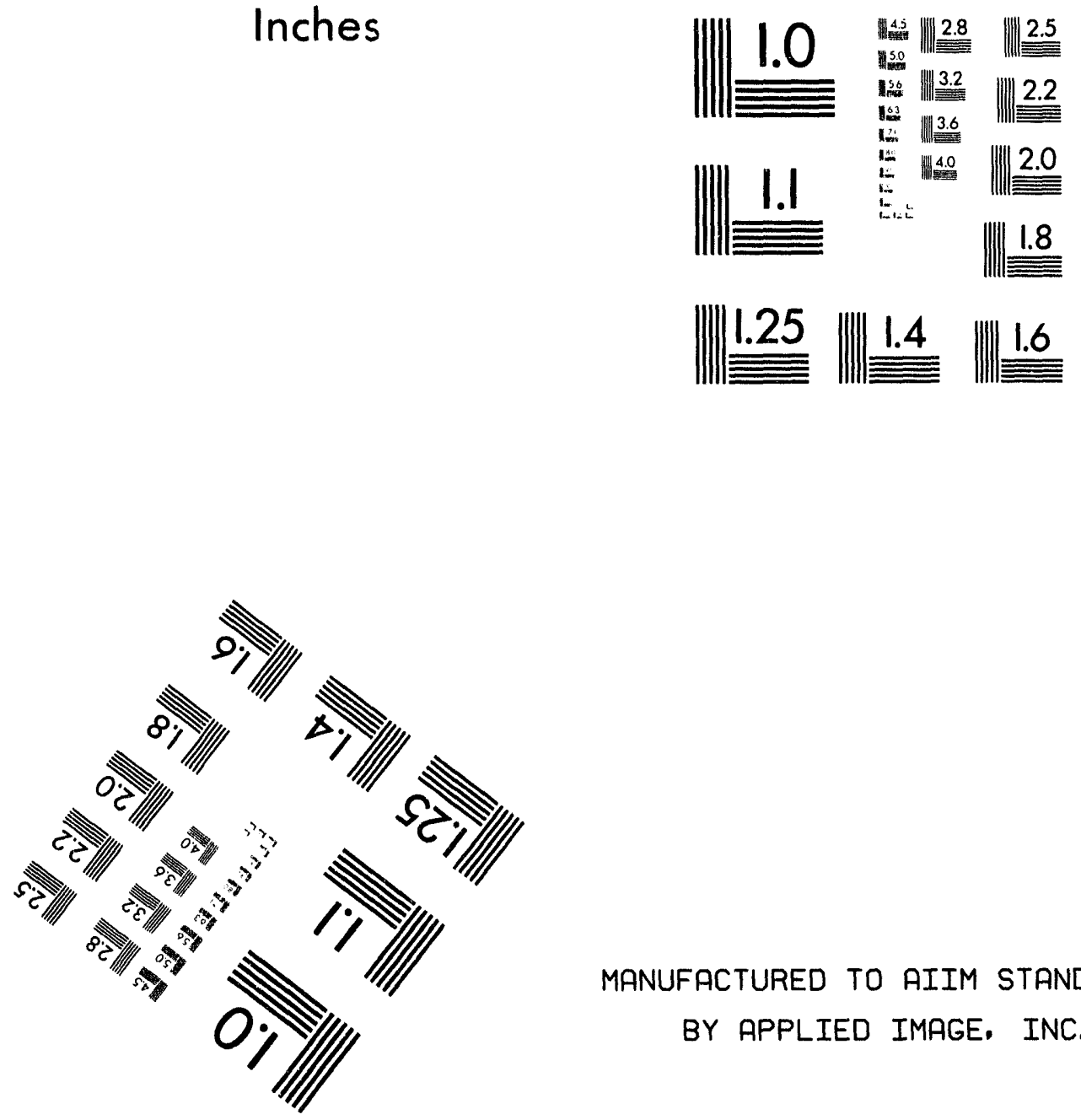

MANUFACTURED TO AIIM STANDARDS BY APPLIED IMAGE. INC.

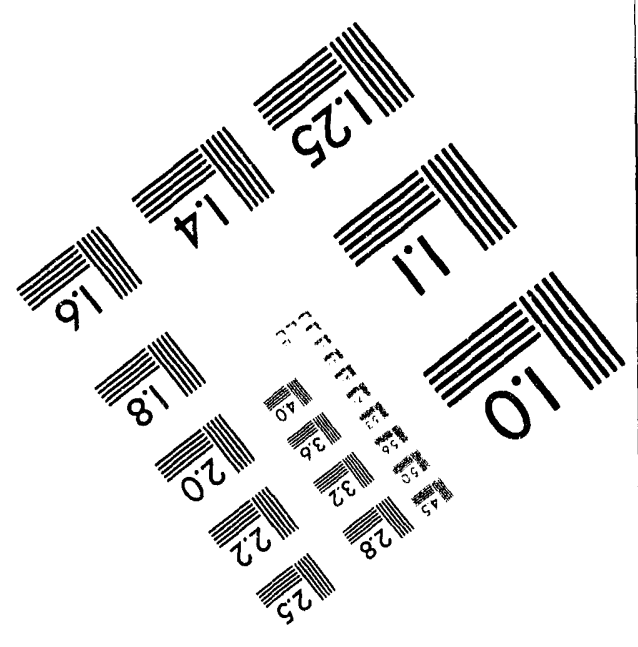




\title{
Alpha particle effects on the internal kink and fishbone modes
}

\author{
Yanlin Wu, C. Z. Cheng, and R. B. White \\ Plasma Physics Laboratory, Princeton University \\ P.O. Box 451 \\ Princeton, New Jersey 08540 USA
}

\begin{abstract}
The effects of alpha particles on the internal kink and fishbone modes are studied analytically. The nonadiabatic contribution from untrapped alpha particles is negligible. Finite inverse aspect ratio, plisma $\beta$ and plasma shaping effects can significantly enhance the trapped particle drift reversal domain in the pitch angle space and reduce the bounceaveraged magnetic drift frequency. The drift reversal effect on the ideal kink mode is small, but the $\beta_{\alpha}$ threshold for the fishbone mode can be much lower than previously predicted. ${ }^{1}$ Moreover, the fishbone mode could be excited by alpha particles even when the plasma is stable in the ideal MHD limit. In addition, the ion diamagnetic drift frequency (finite ion Larmor radius effect) has a strong destabilizing effect on the fishbone mode when it is comparable with the trapped alpha averaged precessional drift frequency, even though it stabilizes the plasma in the ideal MHD limit.
\end{abstract}




\section{Introduction}

The effects of energetic particles on the ideal magnetohydrodynamic (MHD) kink and fishbone modes have been studied extensively. ${ }^{1-8}$ Most previous works were focused on circular cross section tokamaks with zero plasma beta and assumed a narrow pitch angle distribution. In the low-frequency limit, i.e., the wave frequency $\omega$ is well below the precessional frequency $\omega_{d}$ and the diamagnetic drift frequency $\omega_{*}$, the sign of the trapped particle potential energy is proportional to $\omega_{*} / \omega_{d}$ and the energetic trapped particles have a stabilizing effect if $\omega_{*} / \omega_{d}>0$ and destabilizing otherwise. When the hot particle beta $\beta_{h}$ is larger than a critical value, the fishbone mode is excited with $\omega \sim \omega_{d}$. For fusion $\alpha$ particles, however, due to their uniform pitch angle distribution, $\omega_{* \alpha} /\left(\omega_{d}-\omega\right)$ may change from being positive to negative as the particle pitch angle $\lambda$ changes from a deeply trapped to a barely trapped state. The inverse aspect ratio $\varepsilon_{a}$, finite $\beta$ and plasma shaping effects can enlarge the drift reversal domain. Thus the net effect of trapped $\alpha$-particles must be integrated over the entire trapped $\alpha$-particle population. Furthermore, since the trapped $\alpha$ population averaged precessional drift frequency $\left\langle\omega_{d}\right\rangle$ may be comparable with the mode growth rate $\gamma$ or even change sign, some of the previous conclusions ${ }^{1-6}$ based on zero beta, large aspect ratio equilibrium will be greatly modified.

In this paper, we investigate the $\alpha$-particle effects on the internal kink as well as fishbone modes due to effects of plasma $\beta$, plasma shaping, and the core ion finite Larmor radius effect through the diamagnetic drift frequency. The effects of plasma $\beta$, plasma shaping and finite aspect ratio are included in calculating $\omega_{d}$, and both the trapped and passing particle contributions are included in the kinetic potential energy. We find that the stable domain in the $\left(\beta_{\alpha}, \gamma_{b}\right)$ plane can be significantly reduced due to these effects from that previously predicted. ${ }^{1}$ 
In Section II we briefly review an analytical equilibrium solution based on a low beta ordering. ${ }^{9,10}$ The plasma shaping effect is included. An analytical expression of the trapped particle precessional drift frequency is presented in Sec.III. The dispersion relation for the internal kink and fishbone modes is presented in Sec.IV and an analytical marginal stability analysis is given in Sec.V. Numerical results of the dispersion relation are presented in Sec.VI. A ccnclusion is given in Sec.VII.

\section{The shaped equilibrium}

Consider an axisymmetric toroidal plasma, consisting of a core (c) isotropic component and $\alpha$-particles $(\alpha)$ with $n_{\alpha} \ll n_{c}$ and $T_{\alpha} \gg T_{c}$, but $\beta_{\alpha} \ll \beta_{c}$. We consider a transformation ${ }^{9,10}$ from the cylindrical coordinate system $(R, Z, \phi)$ to a flux coordinate system $(\rho, \omega, \psi)$, where $\rho$ labels magnetic surfaces and $\omega$ is a poloidal angle, i. e.

$$
R=R(\rho, \omega), \quad Z=Z(\rho, \omega) .
$$

The transformation leads to a metric tensor $g_{i j}$ defined by the element of length

$$
(d \ell)^{2}=g_{\rho \rho}(d \rho)^{2}+2 g_{\rho \omega} d \rho d \omega+g_{\omega \omega}(d \omega)^{2}+R^{2}(d \phi)^{2}
$$

where

$$
g_{i j}=\partial_{i} R \partial_{j} R+\partial_{i} Z \partial_{j} Z
$$

and the Jacobean $\mathcal{J}=R \sqrt{\operatorname{det} g_{i j}}$ is given by

$$
\mathcal{J}=R\left(\partial_{\omega} R \partial_{\rho} Z-\partial_{\rho} R \partial_{\omega} Z\right)
$$

The magnetic field $\vec{B}$ is represented in the form

$$
\vec{B}=R_{0} B_{0}(f(\rho) \nabla \phi \times \nabla \rho+g(\rho) \nabla \phi)
$$


where $R_{0}$ is the major axis of the torus, $B_{0}$ is the vacuum toroidal magnetic field, and $f$ and $g$ are functions of $\rho$ only. The safety factor $q$ is then given by

$$
q(\rho)=\frac{g(\rho)}{2 \pi f(\rho)} \int_{0}^{2 \pi} \frac{d \omega \mathcal{J}}{R^{2}}
$$

and the equilibrium equation reads

$$
\frac{f}{\mathcal{J}}\left[\partial_{\rho} \frac{f g_{\omega \omega}}{\mathcal{J}}-\partial_{\omega} \frac{f g_{\rho \omega}}{\mathcal{J}}\right]+\frac{p^{\prime}}{R_{0}^{2} B_{0}^{2}}+\frac{g g^{\prime}}{R^{2}}=0
$$

where the pressure $p$ is a function of $\rho$ only, and primes denote derivatives with respect to $\rho$.

Employing a standard low beta tokamak ordering ${ }^{9}$ with $p / B_{0}^{2} \sim \varepsilon^{2}, f \sim \varepsilon$ and $g \sim 1$, equation (7) implies that $g=1+\varepsilon^{2} g_{2}$ where the subscript denotes the order in inverse aspect ratio $\varepsilon$. We seek a solution for the equilibrium furfaces in the form

$$
\begin{gathered}
R=R_{0}-\varepsilon \rho \cos \omega-\varepsilon^{2} \Delta(\rho)+\varepsilon^{2} \sum_{n} s_{n}(\rho) \cos (n-1) \omega+\varepsilon^{3} P \cos \omega+\ldots, \\
Z=\varepsilon \rho \sin \omega+\varepsilon^{2} \sum_{n} s_{n}(\rho) \sin (n-1) \omega-\varepsilon^{3} P \cos \omega+\ldots
\end{gathered}
$$

Substitution of these expressions into the equilibrium equation (7) and normalization of length to $R_{0}$ yield equations for the Shafranov shift $\Delta$, the imposed shaping functions $S_{n}$, and $P$ which corresponds to a relabelling of the surfaces. Thus, we obtain from the coefficients of different harmonics of $\omega$ in each order:

$$
\begin{gathered}
\frac{p_{2}^{\prime}}{B_{0}^{2}}+g_{2}^{\prime}+\frac{f_{1}}{\rho}\left(\rho f_{1}\right)^{\prime}=0 \\
\Delta^{\prime \prime}+\left(\frac{2\left(\rho f_{1}\right)^{\prime}}{\rho f_{1}}-\frac{1}{\rho}\right) \Delta^{\prime}-\frac{2\left(\rho f_{1}\right)^{\prime}}{f_{1}}-1-\frac{2 \rho g_{2}^{\prime}}{f_{1}^{2}}=0
\end{gathered}
$$




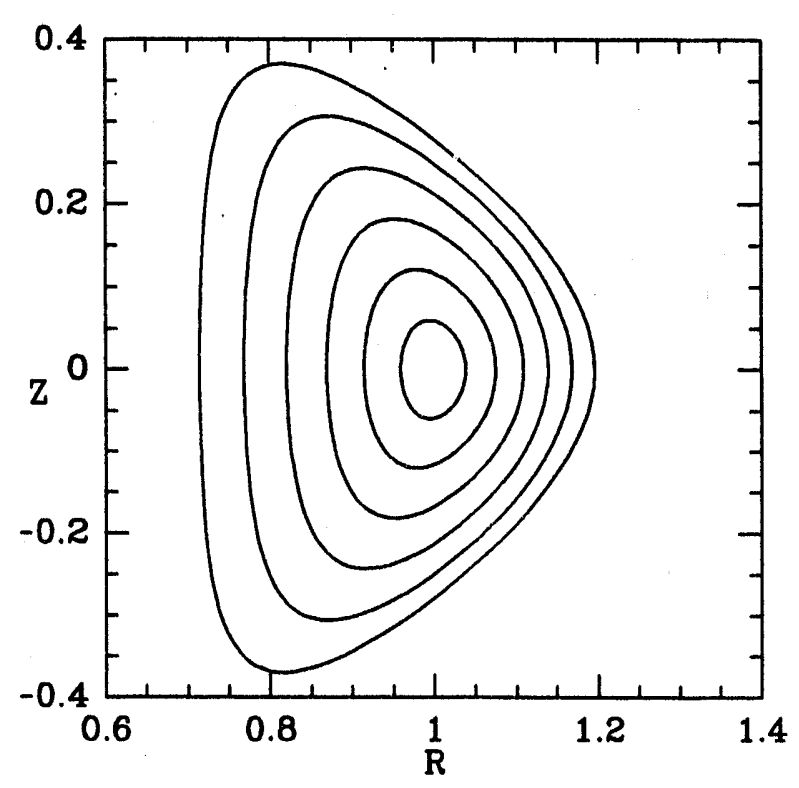

Figure 1: The flux contour plot in R-Z plane.

and

$$
s_{n}^{\prime \prime}+\left(\frac{2\left(\rho f_{1}\right)^{\prime}}{\rho f_{1}}-\frac{1}{\rho}\right) s_{n}^{\prime}-\left(n^{2}-1\right) \frac{s_{n}}{\rho^{2}}=0 .
$$

Note that equation (12) is not driven by the plasma pressure and requires an external origin; it describes how shaping at the plasma surface propagates into the plasma. The ellipticity and triangularity are described by $s_{2}$ and $s_{3}$, respectively with ellipticity $\kappa=$ $\left(1+s_{2}^{\prime}(a)\right) /\left(1-s_{2}^{\prime}(a)\right)$ and trangularity $\delta=s_{3}^{\prime}(a)$. We will assume $s_{n}=0$ for $n \geq 4$ for simplicity. Clearly if $q$ is a constant which corresponds to a flat current profile, $f \sim \rho$ and we have

$$
s_{n}(\rho)=\left(\frac{\rho}{a}\right)^{n-1} s_{n}(a)
$$

In principle, for given $p$ and $q$ or $f$ profiles we can determine all other quantities through 
the above set of equations. For example,

$$
\Delta^{\prime}=\frac{1}{\rho f_{1}^{2}} \int_{0}^{\rho}\left(f_{1}^{2}-\frac{2 \rho p^{\prime}}{B_{0}^{2}}\right) \rho d \rho
$$

and

$$
g_{2}^{\prime}=-\frac{p_{2}^{\prime}}{B_{0}^{2}}-\frac{f_{1}}{\rho}\left(\rho f_{1}\right)^{\prime}
$$

However, since we are only interested in the region within the $q\left(\rho_{s}\right)=1$ surface, i.e. $\rho \leq \rho_{s}$, we will retain only the lowest order solutions. Thus, $\alpha=-p^{\prime} /\left(2 B_{0}^{2} \rho\right), s_{2}=(\kappa-1) /(\kappa+1) \rho$, $s_{3}=\delta \rho^{2} /(2 a), \Delta^{\prime}=(\alpha+1 / 4) \rho$ and $g_{2}^{\prime}=2(\alpha-1) \rho$. The $q$-profile is chosen as

$$
q=q_{0}+\left(1-q_{0}\right)\left(\frac{\rho}{\rho_{s}}\right)^{\ell}
$$

where $q_{0}=q(\rho=0)$ and $\rho_{s}=\rho(q=1)$.

\section{Bounce-averaged magnetic drift frequency}

The toroidal precessional drift frequency of a trapped particle can be expressed $a^{2}$ :

$$
\omega_{d}=\frac{1}{e_{\alpha}} \frac{\partial_{\Psi} J}{\partial_{E} J}
$$

where the longitudinal invariant $J=\oint v_{\|} d \ell$ involves an integral over the trapped particle

orbit. The parallel velocity is $v_{\|}=\sqrt{2(E-\mu B) / M_{\alpha}}, E=M_{\alpha} v^{2} / 2$ is the energy and $\mu$ is the magnetic moment. Here $M_{\alpha}$ and $e_{\alpha}$ are alpha particle mass and charge respectively.

To first order in the inverse aspect ratio $\varepsilon$, we find

$$
\omega_{d}=\omega_{d 0} H(\kappa, \delta, \lambda, \rho) \frac{\lambda \rho_{s}}{\rho}
$$

where

$$
\omega_{d 0}=\frac{q E}{\rho_{s}}
$$


is the deeply trapped particle precessional drift frequency evaluated at the $q=1$ surface, and

$$
H=\left\{\frac{2}{1+\kappa} G_{1}+2 \hat{s} G_{2}-\Delta^{\prime}\left(1+2 G_{3}+G_{4}\right)-2 \rho\left(\alpha-1+G_{4}\right)-\frac{\delta \rho}{a} G_{5}\right\}\left\{1-\Delta^{\prime} G_{1}\right\}^{-1}
$$

with

$$
\begin{gathered}
K\left(k^{2}\right)=\int_{0}^{\theta_{b}} \frac{d \theta}{\sqrt{2\left(\cos \theta-\cos \theta_{b}\right)}} \\
G_{1}=K^{-1} \int_{0}^{\theta_{b}} \frac{\cos \theta}{\sqrt{2\left(\cos \theta-\cos \theta_{b}\right)}} d \theta=2 \frac{E}{K}-1, \\
G_{2}=K^{-1} \int_{0}^{\theta_{b}} \sqrt{2\left(\cos \theta-\cos \theta_{b}\right)} d \theta=2\left\{\frac{E}{K}-\left(1-k^{2}\right)\right\}, \\
G_{3}=K^{-1} \int_{0}^{\theta_{b}} \sqrt{\left(\cos \theta-\cos \theta_{b}\right) / 2} \cos \theta d \theta=\frac{2}{3}\left\{\left(1-k^{2}\right)+\left(2 k^{2}-1\right) \frac{E}{K}\right\}, \\
G_{4}=K^{-1} \int_{0}^{\theta_{b}} \frac{\cos { }^{2} \theta}{\sqrt{2\left(\cos \theta-\cos \theta_{b}\right)}} d \theta=\frac{1}{3}\left\{1+2\left(1-2 k^{2}\right) G_{1}\right\}, \\
G_{5}=K^{-1} \int_{0}^{\theta_{b}} \frac{\cos 2 \theta}{\sqrt{2\left(\cos \theta-\cos \theta_{b}\right)}} d \theta=2 G_{4}-1, \\
\hat{s}=\frac{\rho}{q} \partial_{\rho} q .
\end{gathered}
$$

In the above expressions $\theta_{b}$ is the bounce angle, $k^{2}=\sin ^{2}\left(\theta_{b} / 2\right)=(1+\rho-\lambda) /(2 \rho), K\left(k^{2}\right)$ and $E\left(k^{2}\right)$ are the first and second complete elliptic integrals respectively, $\lambda=\mu B_{0} / E$ is the pitch angle, and $\rho_{s}=\rho(q=1)$. For trapped particles we have $0 \leq k^{2} \leq 1$. 


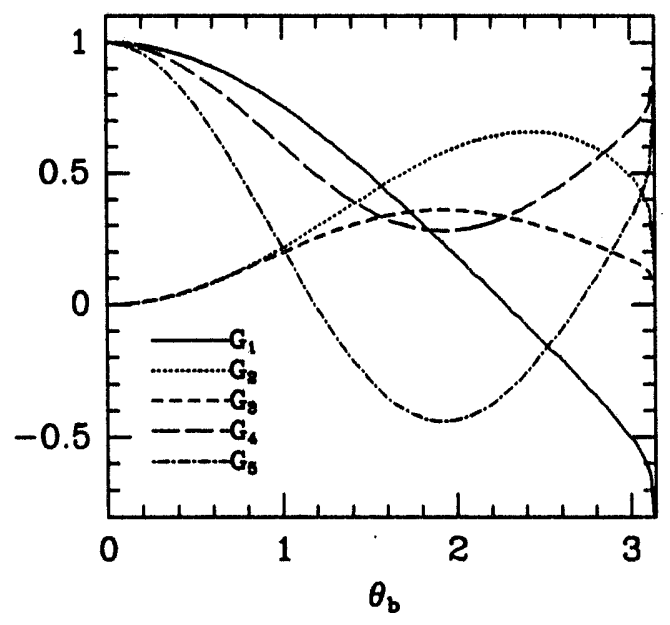

Figure 2: The variations of function $G$ with the trapped particle bounce angle $\theta_{b}$ for $\varepsilon=0.1$.

The effect of ellipticity $\kappa$ is mainly to reduce the magnitude of the precessional drift frequency while the triangularity $\delta$, and plasma $\beta$ will increase the drift reversal domain in the pitch angle space. The finite $\beta$ effect is very important when the plasma $\beta$ approaches the ballooning limit in which case $\beta \sim \varepsilon$ and $\delta \sim O(1)$. Note that in the previous calculations of $\alpha$-particle effect on the internal kink mode,$^{1}$ the effects due to finite $\beta$, plasma shape, and inverse aspect ratio corrections were not included, and $\omega_{d}=\omega_{d 0} G_{1} \rho_{s} / \rho$ was used.

Fig. 3 compares the dependence of $H$ as a function of the bounce angle $\theta_{b}$ for the zero beta circular plasma case and a general finite beta case with $\kappa=1.5, \delta=0.5, \hat{s}=0.4, \alpha=$ 0.75 , and $\rho=\rho_{s}=0.1$. The effect of $\kappa$ is to reduce the magnitude of $\omega_{d}$, and the effects of $\alpha$ and $\delta$ are to increase the drift reversal domain.

Figure 4 plots the trapped particle domain in $(\rho, \lambda)$ space and the $\omega_{d}=0$ curve (Solid curve). For a given radial location, $\omega_{d}$ changes from being positive to negative as pitch $\lambda$ 


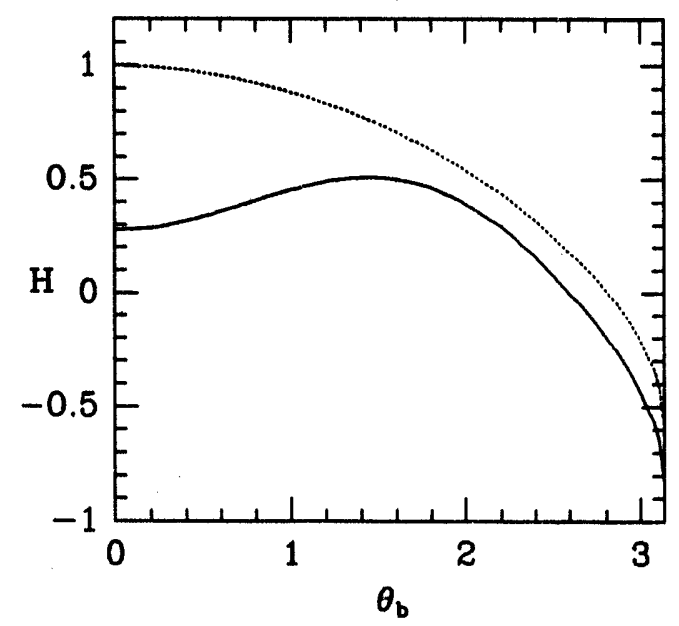

Figure 3: The variations of function $H$ with the trapped particle bounce angle $\theta_{b}$. The solid curve uses the present theory with $\kappa=1.5, \delta=0.5, \hat{s}=0.4, \alpha=0.75$ and $\rho=\rho_{s}=0.1$ and the doted curve corresponds to a zero beta, circular plasma. 


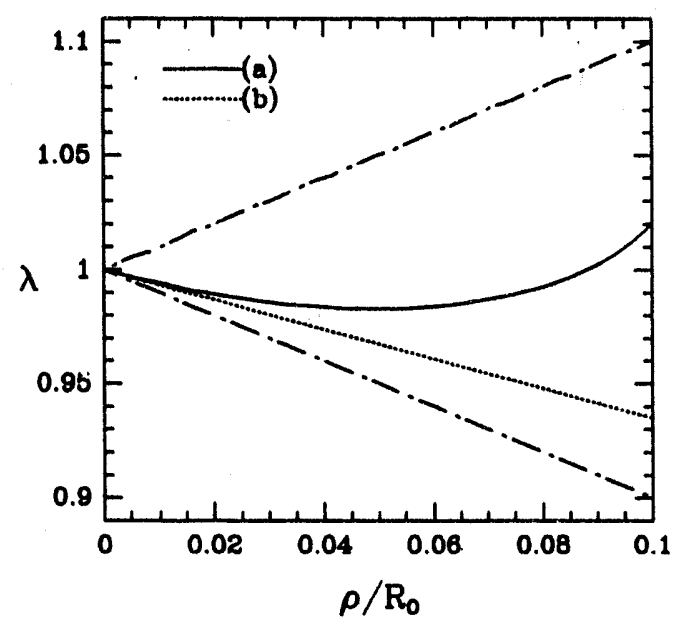

Figure 4: The $\omega_{d}=0$ curves in the trapped particle $(r, \lambda)$ domain. Curve (a) uses the present $\omega_{d}=\omega_{d 0} g$ with $\kappa=1.5, \delta=0.3$ and $\alpha=0.5$, and curve (b) uses $\omega_{d}=\omega_{d 0} \frac{\rho_{e}}{\rho} G_{1}$.

decreases from a deeply trapped state to a barely trapped state, which means that $\omega_{*} / \omega_{d}$ also varies from being positive to negative. If the trapped particles are destabilizing to a certain MHD mode for $\omega_{*} / \omega_{d}>0$ in certain pitch angle region, the trapped particles in the pitch angle region with $\omega_{*} / \omega_{d}<0$ would be stabilizing. Thus, the net effect of trapped $\alpha$-particles must be integrated over the entire trapped $\alpha$-particle population. If the drift reversal domain is large enough so that the net contribution from the term $\omega_{*} / \omega_{d}$ is negative, then the ideally MHD stable kink mode can be destabilized by the $\alpha$-particles. Also shown in Fig. 4 is the $\omega_{d}=0$ curve (Dashed line) without the finite inverse aspect ratio $\varepsilon$, shaping factors $\kappa$ and $\delta$, and plasma $\beta$ effects. It is clear that the drift reversal domain increases due to these corrections. 


\section{Dispersion relation}

In discussing stability it is convenient to use the coordinate $(r, \theta)$ rather than $(\rho, \omega)$, where $^{11}$

$$
r^{2}=2 R_{0} \int_{0}^{\rho} d \rho \int \frac{d \omega \mathcal{J}}{R^{2}}, \quad \theta=2 \pi \frac{\int^{\omega} \frac{d \omega \mathcal{J}}{R^{2}}}{\int_{0}^{2 \pi} \frac{d \omega \mathcal{J}}{R^{2}}}
$$

To second order in $\varepsilon$, we have

$$
r=\rho+O\left(\varepsilon^{3}\right)
$$

and

$$
\theta=\omega+\left(\Delta^{\prime}+\frac{r}{R_{0}}\right) \sin \omega+O\left(\varepsilon^{2}\right)
$$

In this coordinate system, the volume element is

$$
d^{3} \mathbf{x}=2 \pi R_{0} r\left\{1-\left(r+\Delta^{\prime}\right) \cos \theta\right\} d r d \theta
$$

The kinetic-MHD dispersion relation after division by the constant $2 \pi R\left(\frac{r_{,} B}{2 R}\right)^{2}\left|\xi_{0}\right|^{2}$ has the following quadratic form ${ }^{4,12,8}$

$$
D(\omega)=\delta W_{f}+\delta W_{k}-\delta K=0
$$

where

$$
\delta K=i \frac{\sqrt{\omega\left(\omega-\omega_{* i}\right)}}{\tilde{\omega}_{A}}
$$

is the inertial energy, $\tilde{\omega}_{A}=v_{A} /(\sqrt{3} \hat{s} R), \hat{s}=r_{s} q_{s}^{\prime}, q\left(r_{s}\right)=1$, and

$$
\delta W_{f}=\ell\left(1-q_{0}\right)\left\{\frac{13}{16(\ell+4)}-\frac{3 \beta_{p}^{2}}{4-\ell}-\frac{12 \ell^{2}\left(1-q_{0}\right)^{2} s_{2}^{2}\left(r_{s}\right)}{(4+\ell)^{2}(6-\ell)} \frac{R_{0}^{2}}{r_{s}^{4}}+\frac{12 s_{3}^{2}\left(r_{s}\right)}{4+\ell} \frac{R_{0}^{2}}{r_{s}^{4}}\right\}
$$


is the fluid potential energy due to the core components.

The energetic particle potential energy $\delta W_{k}$ has two parts: one is the adiabatic part $\delta W_{k}^{(a)}$ and the other is the non-adiabatic part $\delta W_{k}^{(n a)}$. The adiabatic contribution due to both trapped and passing particles is independent of the mode frequency and is given by

$$
\begin{gathered}
\delta W_{k}^{(a)}=-\frac{3}{4 r_{s}^{2}} \frac{\Omega_{\alpha}}{E_{\alpha}} \int_{0}^{r_{s}} r^{2}\left(r+\Delta^{\prime}\right) \omega_{* \alpha} \beta_{\alpha} d r \\
=-\frac{3}{4 r_{s}^{3}} \int_{0}^{r_{s}} r^{2}\left(r+\Delta^{\prime}\right) \beta_{\alpha} \frac{\omega_{* \alpha}}{\omega_{d 0}} d r .
\end{gathered}
$$

Since the plasma equilibrium is determined by the total plasma pressure, we will combine the $\delta W_{k}^{(a)}$ term (which is from $\vec{\xi}_{\perp} \cdot \nabla P_{\alpha}$ ) with the core plasma $\delta \hat{W}_{f}$ (which is from $\vec{\xi}_{\perp} \cdot \nabla P_{c}$ ) for convenience.

The non-adiabatic part due to the trapped $\alpha$-particles is given by

$$
\begin{gathered}
\delta W_{k, t}^{(n a)}=\frac{2^{13 / 2} \pi^{2} M_{\alpha} R^{2}}{B^{2} r_{s}^{2} \xi_{0}^{2}} \int_{0}^{r_{s}} r d r \int_{1-r}^{1+r} d \lambda \\
\times \int_{0}^{\infty} d E \frac{E^{5 / 2} k_{b}}{\omega_{d}-\omega}\left(\omega \partial_{E} F-\frac{1}{\Omega_{c} r} \partial_{r} F\right)\left(\frac{\xi_{0} k_{2}}{2 R}\right)^{2},
\end{gathered}
$$

where

$$
k_{b}=\frac{1}{2 \pi} \oint \frac{d \theta}{\sqrt{1-\lambda / h}}=\frac{2}{\pi} \sqrt{\frac{2}{r \lambda}} K\left(k^{2}\right)
$$

and

$$
k_{2}=\langle\cos (q \theta)\rangle=G_{1}-(q-1) G_{2}
$$

We assume a slowing-down distribution function with uniform pitch, i.e.,

$$
F(r, E, \lambda)= \begin{cases}c n(r) E^{-3 / 2}, & \text { if } E \leq E_{\alpha} \\ 0, & \text { otherwise }\end{cases}
$$


and the normalization factor $c$ is determined through the hot component pressure

$$
p_{\alpha}(r)=\int_{0}^{E_{\alpha}} F M_{\alpha} E d^{3} \mathbf{v}
$$

which gives

$$
c=\frac{p_{\alpha}}{4 \sqrt{2} \pi M_{\alpha} n(r) E_{\alpha}} .
$$

We find

$$
\delta W_{k, t}^{(n a)}=\frac{3}{4 r_{s}^{2}} \int_{0}^{r_{s}} r \beta_{\alpha}(r) d r \int_{1-r}^{1+r} d \lambda \frac{k_{2}^{2}}{k_{b}}\left\{\frac{\omega_{* \alpha}}{\omega_{d}}+\frac{\omega}{\omega_{d}}\left(\frac{\omega_{* \alpha}}{\omega_{d}}-1\right) S\left(\frac{\omega_{d}}{\omega}\right)\right\}
$$

where

$$
\omega_{* \alpha}=-\frac{2}{3} \frac{E_{\alpha}}{\Omega_{\alpha} r} \partial_{r} \ln n(r)
$$

is the $\alpha$-particle diamagnetic frequency,

$$
\beta_{\alpha}(r)=\frac{8 \pi p_{\alpha}(r)}{B^{2}}
$$

is the local trapped $\alpha$-particle $\beta$, and

$$
S\left(\frac{\omega_{d}}{\omega}\right)= \begin{cases}\ln \left|1-\frac{\omega_{d}}{\omega}\right|+\pi i, & \text { if } \gamma=0 \text { and } 0<\frac{\omega_{r}}{\omega_{d}} \leq 1 \\ \ln \left(1-\frac{\omega_{d}}{\omega}\right)+2 \pi i, & \text { if } \frac{\gamma}{\omega_{d}}<0 \text { and } 0<\frac{\omega_{r}}{\omega_{d}} \leq 1 \\ \ln \left(1-\frac{\omega_{d}}{\omega}\right), & \text { otherwise. }\end{cases}
$$

Note if $\omega_{d} \ll \omega$, the singularity is removed by using the analytic expression

$$
\frac{\omega_{* \alpha}}{\omega_{d}}+\frac{\omega}{\omega_{d}}\left(\frac{\omega_{* \alpha}}{\omega_{d}}-1\right) S\left(\frac{\omega_{d}}{\omega}\right) \simeq 1-\frac{\omega_{* \alpha}-\omega_{d}}{2 \omega}
$$

The non-adiabatic part of the kinetic potential energy due to the circulating $\alpha$-particles is given by

$$
\delta W_{k, c}^{(n a)}=\frac{2^{13 / 2} \pi^{2} M_{\alpha}}{B^{2} r_{s}^{2}} \int_{0}^{r_{s}}\left(r+\Delta^{\prime}\right) d r \int_{0}^{1-r} d \lambda
$$




$$
\times \int_{0}^{\infty} d E \frac{\omega E^{5 / 2}}{(1-q)^{2} \omega_{t}^{2}-\omega^{2}}\left(\omega \partial_{E} F-\frac{1}{\Omega_{c} r} \partial_{r} F\right) k_{c}^{2} k_{t}
$$

where

$$
\begin{gathered}
k_{t}=\frac{1}{2 \pi} \oint \frac{d \theta}{\sqrt{1-\lambda / h}}=\frac{1}{\sqrt{1-\lambda}} \\
k_{c}=\left\langle\left(1-\frac{\mu B}{2 E}\right) \cos \theta\right\rangle=-\frac{\lambda^{2} r}{8(1-\lambda)}
\end{gathered}
$$

and

$$
\omega_{t}=\frac{\left\langle v_{\|}\right\rangle}{q R}=\frac{\sqrt{2 E(1-\lambda)}}{q R} .
$$

The energy integral can be carried out and is given by

$$
\begin{aligned}
I= & \int_{0}^{\infty} d E \frac{E^{5 / 2}}{(1-q)^{2} \omega_{t}^{2}-\omega^{2}}\left(\omega \partial_{E} F-\frac{1}{\Omega_{c} r} \partial_{r} F\right) \\
& =\frac{3}{2} \frac{c n E_{\alpha}}{\Xi}\left\{\omega_{* \alpha}+\omega\left(\frac{\omega_{* \alpha} \omega}{\Xi}-1\right) S_{c}\right\},
\end{aligned}
$$

where

$$
\begin{gathered}
\Xi=\omega_{c}^{2}(1-\lambda), \\
\omega_{c}=\frac{1-q}{q R} \sqrt{2 E_{\alpha}},
\end{gathered}
$$

and

$$
S_{c}= \begin{cases}\ln \left|1-\frac{\Xi}{\omega^{2}}\right|+\pi i, & \text { if } \gamma \omega=0 \text { and } 0<\frac{\omega_{x}^{2}-\gamma^{2}}{\Xi} \leq 1 \\ \ln \left(1-\frac{\Xi}{\omega^{2}}\right)+2 \pi i, & \text { if } \frac{\gamma \omega}{\Xi}<0 \text { and } 0<\frac{\omega_{r}^{2}-\gamma^{2}}{\Xi} \leq 1 \\ \ln \left(1-\frac{\Xi}{\omega^{2}}\right), & \text { otherwise. }\end{cases}
$$

Thus the nonadiabatic circulating particle kinetic potential energy is given by

$$
\delta W_{k, c}^{(n a)}=\frac{3 \omega}{64 r_{s}^{2}} \int_{0}^{r_{s}} \frac{\beta_{\alpha} r^{3}}{\omega_{c}^{2}} d r \int_{0}^{1-r} \frac{\lambda^{4}}{(1-\lambda)^{7 / 2}}\left\{\omega_{* \alpha}+\omega\left(\frac{\omega_{* \alpha} \omega}{\Xi}-1\right) S_{c}\right\}
$$




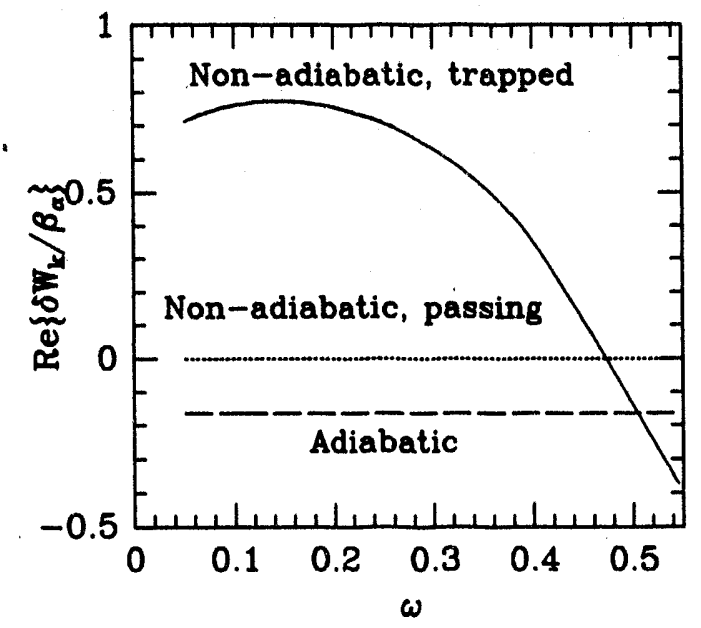

Figure 5: The comparison of the real part of $\delta W_{k}$ due to all components.

Figure 5 compares the nonadiabatic contributions of the energetic trapped and circulating particles as well as the total energetic particle adiabatic contribution. In general the magnitude of $\delta W_{k, c}^{(n a)}$ is much smaller that that of $\delta W_{k, t}^{(n a)}$ and is negligible.

It is convenient to normalize all the quantities to the constant frequency $\omega_{d 0}$, i.e., let $\Delta=\omega_{* \alpha} / \omega_{d 0}, \nu=\tilde{\omega}_{A} / \omega_{d 0}, \Omega=\omega / \omega_{d 0}$. Defining $\gamma_{b}=-\nu\left(\delta \hat{W}_{f}+\delta W_{k}^{(a)}\right)$, the dispersion relation then reduces to

$$
D(\Omega)=-i \sqrt{\Omega(\Omega-\zeta)}-\gamma_{b}+\nu \delta W_{k, t}^{(n a)}=0
$$

\section{Marginal stability analysis}

The marginal stability condition can be understood by simplifying the dispersion relation; the pitch angle integration is approximated with an expression evaluated at an averaged value of precessional drift frequency $\bar{\omega}_{d}$. The effect of magnetic drift reversal is to 
reduce the averaged precessional drift frequency $\bar{\omega}_{d}$. Then the marginal stability conditions for both the ideal kink and the fishbone modes are determined by

$$
\omega\left(1-\beta_{\alpha}^{2} \sigma(\omega)^{2} / \beta_{0}^{2}\right)=\omega_{* i}
$$

and

$$
\gamma_{b}=\frac{\beta_{\alpha}}{\pi \beta_{0}}\left(\bar{\omega}_{d}+\omega \ln \left|1-\frac{\bar{\omega}_{d}}{\omega}\right|\right)
$$

where

$$
\beta_{0}=\frac{4 \bar{\omega}_{d}^{2}}{3 \pi \omega_{* \alpha} \bar{\omega}_{A}} .
$$

Note that at $\beta_{\alpha}=0$, marginal stability for the ideal kink is possible for $\omega_{* i} / 2 \leq \omega_{r} \leq \omega_{* i}$ and $\gamma_{b} \leq \omega_{* i} / 2$

For $\beta_{\alpha} \geq 0$, the real frequency of the ideal kink mode at marginal stability is given by

$$
\omega=\frac{\omega_{* i}}{1-\frac{\beta_{\alpha}^{2} \sigma(\omega)^{2}}{\beta_{0}^{2}}}
$$

and the stability boundary is given by

$$
\gamma_{b}=\frac{3 \omega_{* \alpha} \tilde{\omega}_{A} \beta_{\alpha}}{4 \bar{\omega}_{d}}\left(1+\frac{\omega_{* i}}{\bar{\omega}_{d}} \ln \left|1-\frac{\bar{\omega}_{d}}{\omega_{* i}}\right|\right)
$$

As $\bar{\omega}_{d}$ decreases, the marginal stability boundary curve for the ideal kink mode has a steeper slope in the $\left(\beta_{\alpha}, \gamma_{b}\right)$ space. On the other hand, the contribution of $\omega_{* i}$ is to reduce $\gamma_{b}$ if $\left|1-\bar{\omega}_{d} / \omega_{* i}\right|<1$, and vice versa.

At marginal stability the real frequency of the fishbone mode is much larger than $\omega_{* i}$, but smaller than $\bar{\omega}_{d}$, and in the lowest order is given by the equation

$$
\bar{\omega}_{d}+\omega_{0} \ln \left|1-\frac{\bar{\omega}_{d}}{\omega_{0}}\right|=0
$$


Including the first order correction the frequency is roughly given by

$$
\omega=\omega_{0}\left(1-\frac{0.3 \gamma_{b} \bar{\omega}_{d}}{\beta_{\alpha} \omega_{* \alpha} \tilde{\omega}_{A}}\right)
$$

for

$$
\frac{4 \gamma_{b} \bar{\omega}_{d}}{3 \beta_{\alpha} \omega_{*} \tilde{\omega}_{A}} \ll 1
$$

where $\omega_{0}=0.78 \omega_{d}$. The marginal stability condition is given by

$$
\beta_{\alpha} \sigma(\omega)=\frac{4 \bar{\omega}_{d}^{2}}{3 \pi \omega_{* \alpha} \tilde{\omega}_{A}} \sqrt{1-\frac{1.28 \omega_{* i}}{\bar{\omega}_{d}}\left(1+\frac{0.3 \gamma_{b} \bar{\omega}_{d}}{\beta_{\alpha} \omega_{* \alpha} \tilde{\omega}_{A}}\right)} .
$$

Thus, for the fishbone branch, the effect of reducing $\bar{\omega}_{d}$ or increasing $\omega_{* i}$ is to reduce the critical $\beta_{\alpha}$, and vice versa.

\section{Numerical results}

In the following we present numerical solutions of the dispersion relation, Eq. (54). Consider ITER parameters $E_{\alpha}=3.5 \mathrm{MeV}, E_{i}=20 \mathrm{KeV}, n \sim 10^{14} \mathrm{~cm}^{-3}, B \sim 6 \times$ $10^{4} G, R=800 \mathrm{~cm}, a=300 \mathrm{~cm}, r_{s} \sim a / 3, L_{i}=-\left(\partial_{r} n_{i}\right)^{-1} \sim O(a), L_{\alpha}=-\left(\partial_{r} n_{\alpha}\right)^{-1} \sim$ $O(a / 2)$, and we have: $\Delta \sim O(5), \nu \sim O(20)$. The $q(r)$ profile is chosen to be quadratic. Fig. 6 shows the marginal stability curves in the $\left(\beta_{\alpha}, \gamma_{b}\right)$ space for $\omega_{* i}=0.05$. In the domain under the curves, the plasma is stable; the left boundary is for the ideal kink mode, and the right boundary is for the fishbone mode. The solid curve is obtained by using $\omega_{d}=\omega_{d 0} H r_{s} / r$, and the dotted curve is obtained by using $\omega_{d}=\omega_{d 0} G_{1} r_{s} / r$ so that plasma beta and finite inverse aspect ratio effects are neglected. Here $H<G_{1}$. It is clear that the drift reversal effect is reducing the average $\bar{\omega}_{d}$, and thus the critical $\beta_{\alpha}$ for both the ideal kink and the fishbone modes. The drift reversal has a small stabilizing 


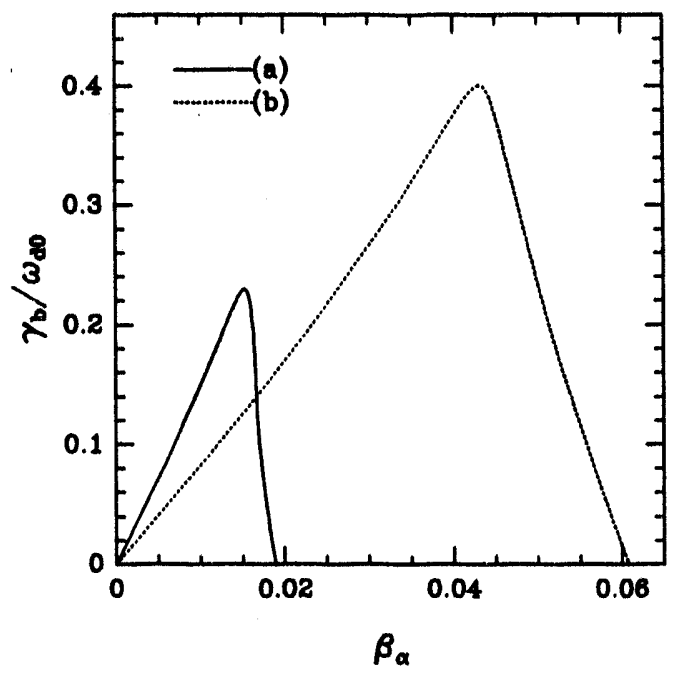

Figure 6: Marginal stability curves in the $\left(\beta_{\alpha}, \gamma_{b}\right)$ plane. Curve (a) uses $\omega_{d}=\omega_{d 0} H r_{s} / r$ with $\omega_{* i}=0.05, \alpha=0.5, \kappa=1.5$ and $\delta=0.3$, and curve (b) uses $\omega_{d}=\omega_{d 0} G_{1} r_{s} / r$.

effect on the ideal kink branch; the slope of the stability curve is proportional to $1 / \bar{\omega}_{d}$ near $\beta_{\alpha}=0$. However, the drift reversal has a large destabilizing effect on the fishbone mode; the threshold for the fishbone is substantially lowered since the critical $\beta_{\alpha} \sim \bar{\omega}_{d}^{2}$. We note that even when the total plasma $\beta$ is below the ideal internal kink threshold, the fishbone mode can be excited with large $\beta_{\alpha}$.

Figures 7 and 8 plot the marginal stability boundaries in the $\left(\beta_{\alpha}, \gamma_{b}\right)$ space for ellipticity $\kappa$ and trangularity $\delta$ dependences. The fixed parameters are the same as in Fig. 6 . In general, as $\kappa$ increases, the magnetic field curvature decreases and so does $\omega_{d}$. It is clear that as $\kappa$ or $\delta$ gets larger, the stable domain becomes narrower mainly due to the destabilizing effect on the fishbone mode. The $\beta_{\alpha}$ threshold for the stabilization of the ideal kink mode is only slightly decreased by $\kappa$ and $\delta$. But, the threshold for the fishbone mode excitation can be significantly reduced by $\kappa$. As pointed out by Connor, ${ }^{10}$ the ellipticity $\kappa$ has a small 


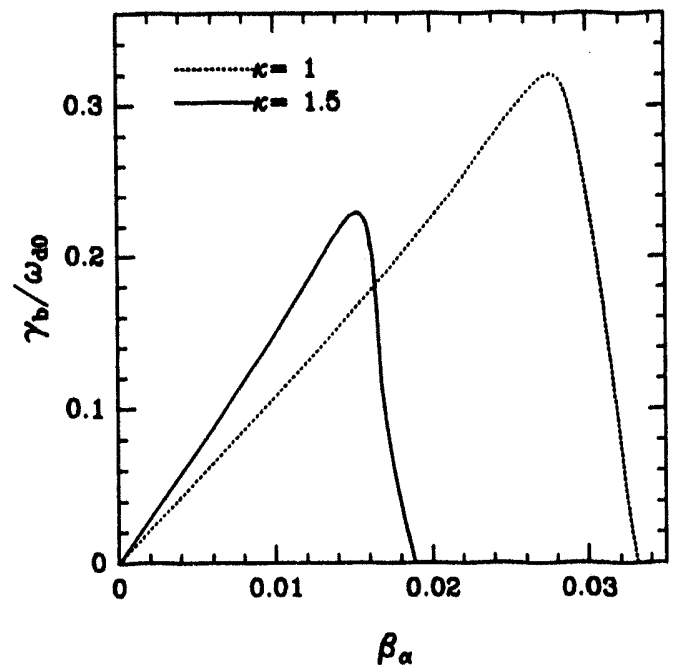

Figure 7: The ellipticity $\kappa$ dependence of the marginal stability curves in the $\left(\beta_{\alpha}, \gamma_{b}\right)$ plane for $\alpha=0.5, \delta=0.3$, and $\omega_{* i}=0.05$

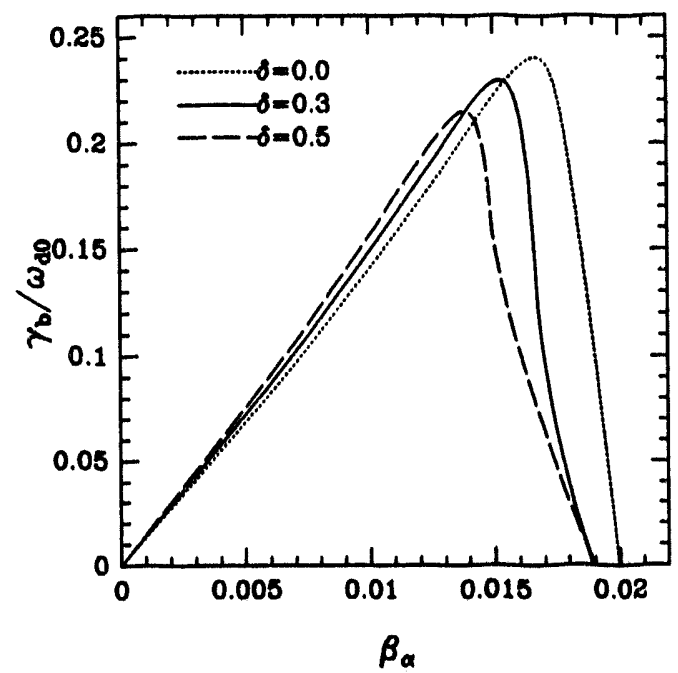

Figure 8: The trangularity $\delta$ dependence of the marginal stability curves in the $\left(\beta_{\alpha}, \gamma_{b}\right)$ plane for $\kappa=1.5, \delta=0.3$ and $\omega_{* i}=0.05$. 


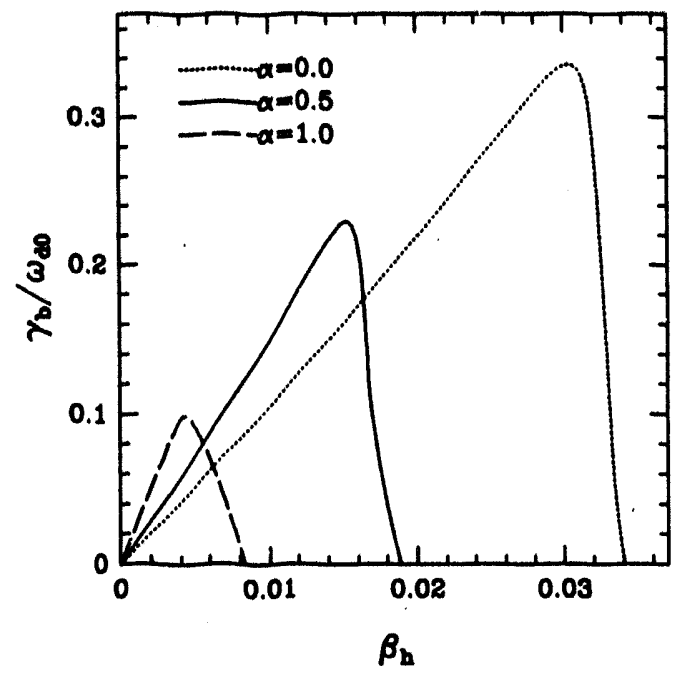

Figure 9: The plasma $\beta$ dependence of the marginal stability curves in the $\left(\beta_{\alpha}, \gamma_{b}\right)$ plane for $\kappa=1.5, \delta=0.3$ and $\omega_{* i}=0.05$.

destabilizing effect but the triangularity $\delta$ has a strong stabilizing effect on the ideal MHD kink growth rate $\gamma_{b}$. Thus, the effect of $\delta$ through $\gamma_{b}$ competes with its destabilizing effect on the fishbone mode.

Figure 9 plots the dependence of the marginal stability boundaries in the $\left(\beta_{\alpha}, \gamma_{b}\right)$ space on plasma $\beta$. The strong destabilizing effect of $\beta$ is mainly due to the appearence of a magnetic well which reduces the drift velocity. The case with $\alpha=0.5$ corresponds to about $2 \%$ average plasma beta for a quardratic pressure profile.

Fig. 10 shows the core ion finite Larmor radius effect (finite $\omega_{* i}$ ) on the stability boundary in the $\left(\beta_{\alpha}, \gamma_{b}\right)$ space. For typical tokamak parameters we expect $\omega_{* i} / \omega_{d 0} \leq 0.05$. As $\omega_{* i}$ increases the $\gamma_{b}$ threshold increases for the ideal kink mode, but decreases much more for the fishbone mode. For the fishbone mode the $\beta_{\alpha}$ threshold is further reduced by $\omega_{* i}$ besides $\omega_{d}$, as predicted from our analytical analysis. The $\omega_{* i}$ effect is very important if 


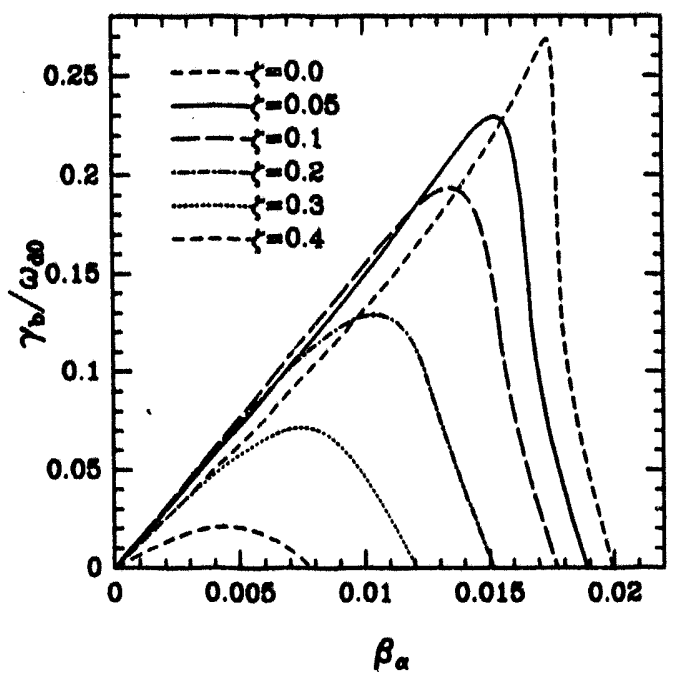

Figure 10: The diamagnetic drift frequency $\zeta\left(=\omega_{* i} / \omega_{d 0}\right)$-dependence of the marginal stability curves in the $\left(\beta_{\alpha}, \gamma_{b}\right)$ plane for $\kappa=1.5, \delta=0.3$ and $\alpha=0.5$.

it is comparable with the averaged magnetic precessional frequency $\omega_{d}$ such as in Neutral Beam Injection cases.

Figure 11 depicts the kink mode frequency $\omega$ and the growth rate $\gamma$ versus $\beta_{h}$ with a fixed ideal kink growth rate $\gamma_{b}$. The stablization of the ideal branch and the destabilization of the fishbone branch due to the fusion $\alpha$-particles is very clear. Moreover, the $\alpha$-particles can still excite the fishbone branch even for $\gamma_{b}<0$, i.e. when kink mode was originally stable in the ideal MHD limit in the absence of $\alpha$-particles. For $\gamma_{b}=0.075, \beta_{\alpha}>0.013$, and the fishbone mode is excited. 

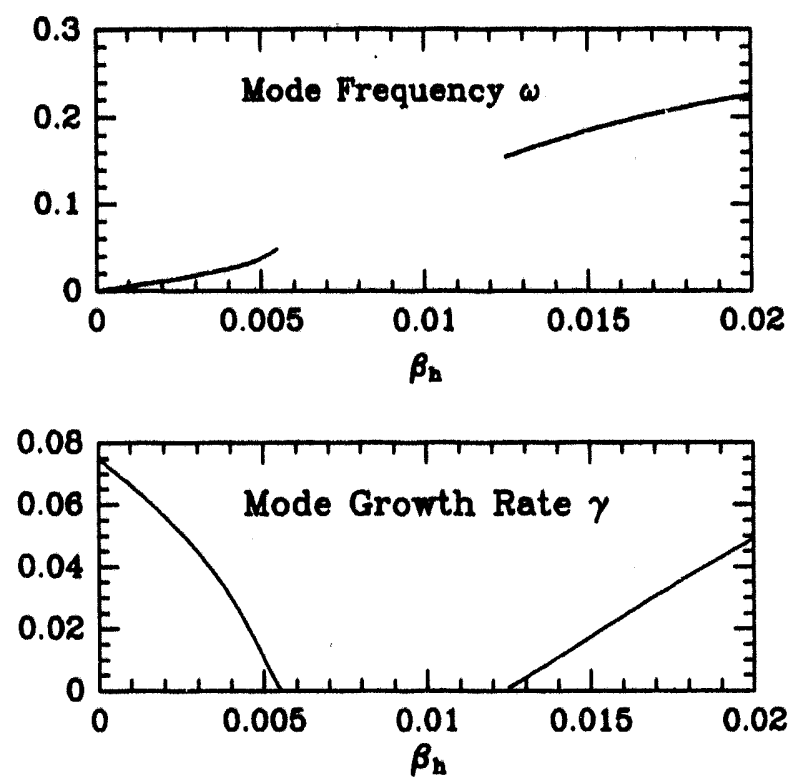

Figure 11: Sample mode frequency and growth rate for $\kappa=1.5, \delta=0$ and $\gamma_{b}=0.075$.

\section{Conclusions}

In summary, the effects of trapped fusion $\alpha$-particles on internal kink and fishbone modes have been investigated. Finite Shafranov Shift, plasma $\beta$, and plasma shaping effects can significantly enhance the trapped particle drift reversal domain in the pitch angle space and reduce the magnitude of the precessional drift frequency. The stable domain for both the ideal kink and the fishbone modes can be significantly reduced. For typical tokamak parameters, the core ion diamagnetic drift effect can narrow the stable domain in the presence of alpha particles, even though it has a stabilizing effect in the ideal MHD limit. 


\section{Acknowledgments}

This work was supported by the United States Department of Energy under contract number DE-AC02-76-CH03073. 


\section{References}

${ }^{1}$ B. Coppi, S. Migliuolo, F. Pegoraro, and F. Porcelli, Phys. Fluids B 2, 927 (1990).

${ }^{2}$ M. N. Rosenbluth and M. L. Sloan, Phys. Fluids 14, 1726 (1971).

${ }^{3}$ M. N. Rosenbluth, S. T. Tsai, J. W. VanDam, and M. G. Engquist, Phys. Rev. Lett. 51, 1967 (1983).

${ }^{4}$ L. Chen, R. B. White, and M. N. Rosenbluth, Phys. Rev. Lett. 52, 1122 (1984).

${ }^{5}$ R. B. White, L. Chen, F. Romenelli, and R. Hay, Phys. Fluids B 28, 278 (1985).

${ }^{6}$ R. B. White, M. N. Bussac, and F. Romanelli, Phys. Rev. Lett. 62, 539 (1989).

${ }^{7}$ C. Z. Cheng, Fusion Technology 18, 443 (1990).

${ }^{8}$ C. Z. Cheng, Phys. Reports 211, 1 (1992).

${ }^{9}$ J. M. Green, J. L. Johnson, and K. E. Weimer, Phys. Fluids 14, 671 (1971).

${ }^{10} \mathrm{~J}$. W. Connor and R. J. Hastie, The effect of shaped plasma cross sections on the ideal internal kink mode in a tokamak, CLM-M106, 1985.

${ }^{11}$ M. N. Bussac, R. Pellat, D. Edery, and J. L. Soule, Phys. Rev. Lett. 35, 1638 (1975).

${ }^{12}$ C. Z. Cheng, Phys. Fluids B 2, 1427 (1990). 


\section{Figure Captions}

Fig. 1 The flux contour plot in R-Z plane.

Fig. 2 The variations of function $G$ with the trapped particle bounce angle $\theta_{b}$ for $\varepsilon=0.1$.

Fig. 3 The variations of function $H$ with the trapped particle bounce angle $\theta_{b}$. The solid curve uses the present iheory with $\kappa=1.5, \delta=0.5, \hat{s}=0.4, \alpha=0.75$ and $\rho=\rho_{s}=0.1$ and the doted curve corresponds to a zero beta, circular plasma.

Fig. 4 The $\omega_{d}=0$ curves in the trapped particle $(r, \lambda)$ domain. Curve (a) uses the present $\omega_{d}=\omega_{d 0} g$ with $\kappa=1.5, \delta=0.3$ and $\alpha=0.5$, and curve (b) uses $\omega_{d}=\omega_{d 0} \frac{\rho_{e}}{\rho} G_{1}$.

Fig. 5 The comparison of the real part of $\delta W_{k}$ due to all components.

Fig. 6 Marginal stability curves in the $\left(\beta_{\alpha}, \gamma_{b}\right)$ plane. Curve (a) uses $\omega_{d}=\omega_{d 0} H r_{s} / r$ with $\omega_{* i}=0.05, \alpha=0.5, \kappa=1.5$ and $\delta=0.3$ and curve (b) uses $\omega_{d}=\omega_{d 0} G_{1} r_{s} / r$.

Fig. 7 The ellipticity $\kappa$ dependence of the marginal stability curves in the $\left(\beta_{\alpha}, \gamma_{b}\right)$ plane for $\alpha=0.5, \delta=0.3$, and $\omega_{* i}=0.05$.

Fig. 8 The trangularity $\delta$ dependence of the marginal stability curves in the $\left(\beta_{\alpha}, \gamma_{b}\right)$ plane for $\kappa=1.5, \delta=0.3$ and $\omega_{* i}=0.05$.

Fig. 9 The plasma $\beta$ dependence of the marginal stability curves in the $\left(\beta_{\alpha}, \gamma_{b}\right)$ plane for $\kappa=1.5, \delta=0.3$ and $\omega_{* i}=0.05$.

Fig. 10 The diamagnetic drift frequency $\zeta\left(=\omega_{* i} / \omega_{d 0}\right)$-dependence of the marginal stability curves in the $\left(\beta_{\alpha}, \gamma_{b}\right)$ plane for $\kappa=1.5, \delta=0.3$ and $\alpha=0.5$.

Fig. 11 Sample mode frequency and growth rate for $\kappa=1.5, \delta=0$ and $\gamma_{b}=0.075$. 

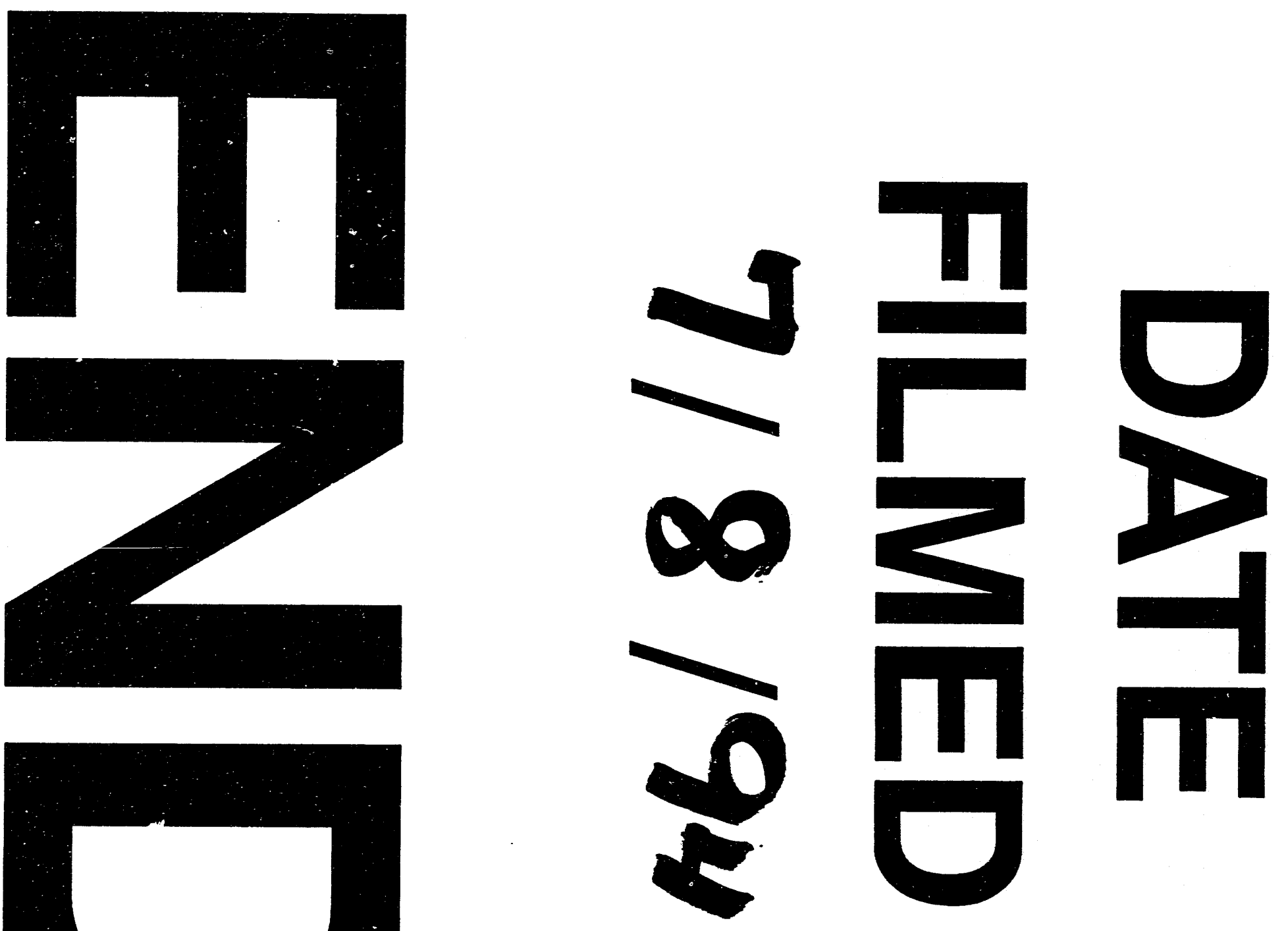

$H$

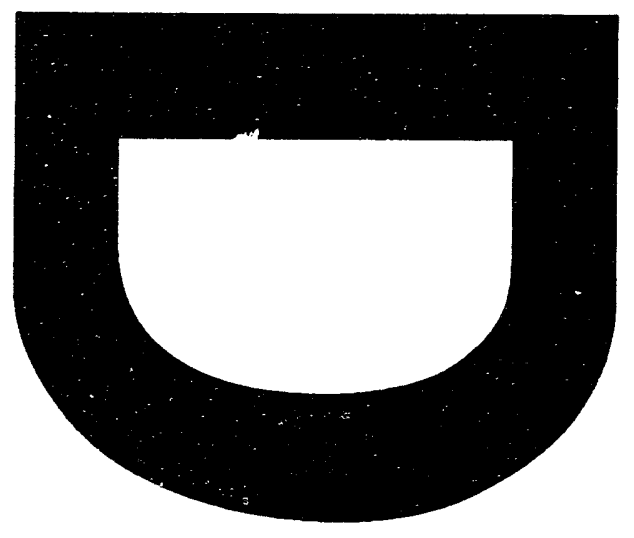


